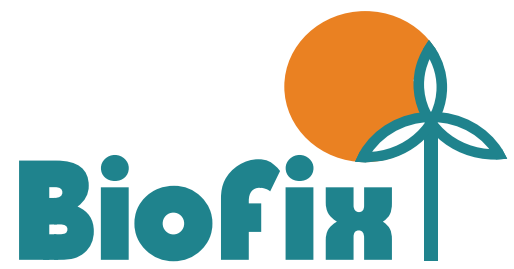

scientific journal

v. 2 n. 22017

\section{POTENCIAL DENDROENERGÉTICO DOS DIFERENTES COMPARTIMENTOS DE Acacia mearnsii CULTIVADA NO ESTADO DO RIO GRANDE DO SUL}

\author{
DENDROENERGETIC POTENTIAL OF DIFFERENT COMPARTMENTS OF \\ Acacia mearnsii CULTURE IN RIO GRANDE DO SUL STATE
}

Recebido em 11/10/2017

Aceito em 17/11/2017

Publicado em 23/11/2017

DOI: dx.doi.org/10.5380/biofix.v2i2.55777

\author{
Dimas Agostinho da Silva \\ Alexandre Behling ${ }^{2}$ \\ Carlos Roberto Sanquetta ${ }^{3}$ \\ Ana Paula Dalla Corte 4 \\ Marieli Sabrina Ruza \\ Heloisa Pscheidt ${ }^{6}$ \\ Sergio Costa Junior ${ }^{7}$
}

Universidade Federal do Paraná, Curitiba, Paraná, Brasil dimas.agostinho.silva@gmail.com ${ }^{1}$, alexandre.behling@yahoo.com.br ${ }^{2}$,carlos_sanquetta@hotmail.com ${ }^{3}$, anapaulacorte@gmail.com ${ }^{4}$,marielisabrinaruza@yahoo.com.br ${ }^{5}$,heloisaa_p@yahoo.com.br ${ }^{6}$ \&o.sergio.costa@gmail.com ${ }^{7}$

\section{RESUMO}

A utilização da biomassa para conversão em energia tem sido um tema muito abordado na atualidade, devido a importância da geração de energia limpa e renovável. Este estudo teve como objetivo analisar o potencial dendroenergético dos compartimentos da Acacia mearnsii De Wild. sob diferentes idades. Para isso, foi conduzido um estudo em dois povoamentos da espécie no estado do Rio Grande do Sul com idades de 1 a 7 anos. A biomassa foi mensurada por compartimento nas diferentes idades e, após esse processo, foi determinado o poder calorífico superior (PCS) das amostras. O cálculo da produtividade energética foi realizado pela multiplicação das variáveis biomassa e PCS. Mediante a análise de variância, evidenciou-se que existem diferenças significativas para o PCS dos compartimentos avaliados, tendo PCS médio para as folhas, a casca, os galhos vivos e a madeira, de 5.408, 4.787, 4.692 e $4.572 \mathrm{kcal} \mathrm{kg}^{-1}$, respectivamente. A produtividade energética da biomassa aumentou com o incremento da idade, com valores variando de $16,3 \mathrm{Gcal} \mathrm{ha}^{-1}$ para o plantio de um ano a $635 \mathrm{Gcal}$ ha $^{-1}$ para o povoamento de sete anos, sendo a madeira, o componente de maior contribuição para esses valores, exceto na primeira idade. Conclui-se que a espécie tem potencial para suprir futuras demandas energéticas e que a produtividade energética varia conforme a idade e o compartimento.

PALAVRAS-CHAVE: Acácia-negra, Biomassa, Dendroenergia.

\section{ABSTRACT}

The use of biomass for conversion to energy has been a subject much approached currently, due to the importance for generation clean and renewable energy. This paper aimed to assess the dendroenergetic potential of the compartments of Acacia mearnsii De Wild. with different ages. A study was conducted in two pure forest stands in Rio Grande do Sul State with ages ranging from 1 to 7 years. Biomass was measured per compartment and age, and Gross Calorific Power (GCP) was assessed through sampling. Energetic productivity was calculated by the product of biomass and GCP variables. It was evidenced by variance analysis that GCP were different between compartments. The average GCP for leaves, bark, live branches, and wood were respectively 5,408, 4,787, 4,692 e 4,572 $\mathrm{kcal} \mathrm{kg}^{-1}$. The energetic biomass productivity increased with increasing age, with values ranging from 16,3 $\mathrm{Gcal} \mathrm{ha}^{-1}$ for stands with one year old to 635 Gcal ha ${ }^{-1}$ for seven years-old. The wood compartment presented the greatest contribution for those values. It was concluded that the species has potential to supply future energetic demands, and that energetic productivity changes with age and compartment.

KEYWORDS: Black-wattle, Biomass, Dendroenergy. 


\section{INTRODUÇÃO}

O uso da biomassa florestal para geração de energia tem sido cada vez mais enfatizado no cenário mundial, uma vez que é uma fonte renovável, limpa e geradora de empregos. No Brasil, a combustão de madeira ou de resíduos é a utilização mais comum da biomassa para atender as várias demandas energéticas, tanto industrial como doméstico e agrícola. Segundo o Ministério de Minas e Energia (BRASIL, 2016), 8,1\% da energia gerada no Brasil provem de lenha e carvão vegetal.

Para aumentar a eficiência da conversão de madeira em energia, é necessária a adoção de técnicas adequadas para avaliação do potencial energético (SILVA et al., 2012). Dessa forma, destaca-se o uso e o estudo da biomassa florestal como uma fonte promissora para a conversão de madeira em energia.

Além de conhecer o estoque de biomassa de uma cultura, é imprescindível conhecer suas propriedades energéticas. Para avaliar o potencial energético de combustíveis formados por biomassa, utiliza-se o poder calorífico superior (PCS), o qual quantifica a energia liberada na combustão completa de uma unidade de massa (PARIKH et al., 2005; BRAND, 2010).

Eloy et al. (2015a) destacam que a participação de produtos advindos da madeira na matriz energética depende do acesso à tecnologia por pequenos e médios produtores, os quais, por exemplo, são detentores de grande parte dos plantios de Acacia mearnsii de Wild no estado do Rio Grande do Sul.

A espécie Acacia mearnsii, é a terceira cultura florestal mais plantada no Brasil (SFB, 2013). Sua madeira é utilizada como fonte energética e para fabricação de celulose e de aglomerados, ao passo que de sua casca é extraído o tanino utilizado na indústria coureira (BYRNE; NAGLE, 1997; CALDEIRA, 2004).

Diante do apresentado, o presente estudo teve por objetivo analisar o potencial dendroenergético dos diferentes compartimentos sob diferentes idades de povoamentos de Acacia mearnsii, tendo em vista a crescente demanda energética proveniente de biomassa vegetal.

\section{MATERIAL E MÉTODOS}

\section{Coleta de dados}

Para a realização deste estudo, utilizaram-se dados de plantios de Acacia mearnsii localizados nos municípios de Piratini e Cristal no estado do Rio Grande do Sul, onde estão concentradas as maiores plantações dessa cultura. O clima da região, segundo classificação de Köppen (KÖPPEN \& GEIGER, 1928), é Cfa mesotérmico subtropical com verões quentes sem estação seca.

As áreas no município de Piratini se encontram sob as coordenadas $31^{\circ} 24^{\prime} \mathrm{S}$ e $52^{\circ} 57^{\prime} \mathrm{W}$ e altitude de aproximadamente $345 \mathrm{~m}$, enquanto as áreas de estudo em Cristal estão localizadas nas coordenadas $30^{\circ} 55^{\prime} \mathrm{S}$ e $52^{\circ} 10^{\prime} \mathrm{W}$ e altitude aproximada de $70 \mathrm{~m}$.

Em cada povoamento, foram delimitadas três unidades amostrais com dimensão de $9 \mathrm{~m} \times 16 \mathrm{~m}$ para as áreas com um ano de idade e espaçamento de $3 \mathrm{~m} \times 1,75$ $\mathrm{m}$; e dimensão de $9 \mathrm{~m} \times 14 \mathrm{~m}$ para os plantios com idades de 3, 5 e 7 anos, com espaçamento de $3 \mathrm{~m} \times 1,5 \mathrm{~m}$. Os tamanhos das parcelas corresponderam a quatro linhas de plantio com dez árvores em cada linha.

Em cada uma das unidades amostrais, os indivíduos tiveram a circunferência à altura do peito (CAP) medida a 1,3 $\mathrm{m}$ do solo mensuradas com fita métrica. Nas mesmas unidades amostrais, foi avaliada a biomassa de duas árvores, as quais apresentavam CAP equivalente à média de cada parcela.

\section{Aferição da biomassa para validação de equações}

A quantificação da biomassa foi calculada individualmente para cada um dos compartimentos da árvore, sendo eles divididos em: madeira do fuste, casca, galhos vivos, galhos mortos e folhas (folhas + flores + frutos).

Para a aferição da biomassa seca do fuste, foram obtidos cinco discos de $2 \mathrm{~cm}$ de espessura nas posições 0\%, 25\%, 50\%, 75\% e $100 \%$ ao longo da altura comercial, considerando o diâmetro de topo de $4 \mathrm{~cm}$. Para os demais compartimentos, foram obtidas amostras com cerca de $200 \mathrm{~g}$, sendo pesadas de imediato para quantificação da massa fresca em balança digital com acurácia de 0,5 g.

Em seguida, as amostras foram secas em estufa de circulação e renovação de $\operatorname{ar}$ a $75^{\circ} \mathrm{C}$. Após o alcance de massa constante, o material foi novamente pesado, propiciando a determinação do teor de umidade e, consequentemente, a biomassa seca por meio da equação (1).

$\mathrm{B}_{\mathrm{i}}=\frac{\mathrm{MU}_{\mathrm{i}} \cdot \mathrm{MS}_{\mathrm{i}}}{\mathrm{MUa}_{\mathrm{i}}}$

Em que: $B_{i}=$ biomassa seca por compartimento $(\mathrm{kg})$; $\mathrm{MU}_{\mathrm{i}}=$ massa fresca por compartimento $(\mathrm{kg}) ; \mathrm{MS}_{\mathrm{i}}=$ massa seca da amostra (kg); e $\mathrm{MUa}_{\mathrm{i}}=$ massa fresca da amostra (kg). 
A biomassa total da árvore foi obtida pela soma da biomassa seca de todos os compartimentos.

\section{Equações para estimativa da biomassa}

Para a estimativa da biomassa, foi utilizado as equações propostas por Dolci et al. (2013), que analisaram equações de biomassa para acácia-negra propostas na literatura para estimar a biomassa do presente conjunto de dados.

\section{Determinação do poder calorífico superior}

Após a secagem das amostras, as mesmas foram reduzidas em moinho Willey e secas novamente em estufa de circulação e renovação do ar a $105^{\circ} \mathrm{C}$. Posteriormente a verificação da massa constante, o poder calorífico superior (PCS) foi analisado para todos os compartimentos, de acordo com a norma ABNT/NBR $8633 / 84$, utilizando uma bomba calorimétrica adiabática (IKA WORKS C5000). Na determinação do PCS, os compartimentos galhos vivos e mortos foram analisados em conjunto.

\section{Determinação da produtividade energética}

A quantidade de energia por hectare, dada pela determinação da produtividade energética (PE), foi obtida em função da biomassa correspondente de cada compartimento (madeira, casca, galho vivo, galho morto e folha), com o respectivo PCS, conforme a equação (2).

$\mathrm{PE}=\mathrm{BS} \cdot \mathrm{PCS}$

Em que: $\mathrm{PE}=$ produtividade energética $\left(\mathrm{Gcal} \mathrm{ha}{ }^{-1}\right)$; $\mathrm{BS}=$ biomassa seca $\left(\mathrm{t} \mathrm{ha} \mathrm{h}^{-1}\right) ;$ e $\mathrm{PCS}=$ poder calorífico superior $\left(\mathrm{kcal} \mathrm{kg}^{-1}\right)$.

\section{Análise dos dados}

Os dados obtidos foram submetidos à análise estatística no software Statistical Analysis System (SAS, 2003), em que se determinou a análise de variância, a análise de regressão e os testes $\mathrm{F}$ e de Tukey a $5 \%$ de probabilidade.

\section{RESULTADOS E DISCUSSÃO}

Análise da altura total, diâmetro à 1,3 $\mathrm{m}$ e sobrevivência
Na Tabela 1 são apresentadas as estatísticas para os valores de diâmetro a 1,3 $\mathrm{m}$ do solo e altura total. Para o diâmetro, as médias encontradas foram de 5,09 cm, 8,15 $\mathrm{cm}, 10,29 \mathrm{~cm}$ e $12,79 \mathrm{~cm}$ para os povoamentos de $1,3,5$ e 7 anos, respectivamente. Os valores de altura total encontrados para a mesma sequência de idade foram $5,77 \mathrm{~m}, 10,50 \mathrm{~m}, 14,09 \mathrm{~m}$ e $16,95 \mathrm{~m}$.

Tabela 1. Estatísticas descritivas da altura total e do diâmetro à 1,3 $\mathrm{m}$ do solo em povoamentos de Acacia mearnsii com idades de 1, 3, 5 e 7 anos

\begin{tabular}{ccccc}
\hline Estatística & Ano 1 & Ano 3 & Ano 5 & Ano 7 \\
\hline \multicolumn{5}{c}{ Diâmetro a 1,3 $\mathbf{m}$ do solo (cm) } \\
\hline Média & 5,09 & 8,15 & 10,29 & 12,79 \\
\hline Erro padrão & 0,09 & 0,16 & 0,24 & 0,26 \\
Desvio padrão & 1,36 & 2,29 & 3,37 & 3,59 \\
Valor mínimo & 1,59 & 1,27 & 2,55 & 3,66 \\
Valor máximo & 8,44 & 13,05 & 17,19 & 21,49 \\
\hline \multicolumn{5}{c}{ Altura total $(\mathbf{m})$} \\
\hline Média & 5,77 & 10,50 & 14,09 & 16,95 \\
\hline Erro padrão & 0,09 & 0,19 & 0,22 & 0,24 \\
Desvio padrão & 1,42 & 2,68 & 3,06 & 3,40 \\
Valor mínimo & 2,80 & 2,40 & 4,10 & 5,60 \\
Valor máximo & 9,10 & 15,20 & 23,00 & 23,80 \\
\hline
\end{tabular}

A porcentagem de sobrevivência decresceu conforme a idade dos cultivos, com valores médios de $91 \%, 86 \%$, $75 \%$ e $65 \%$, respectivamente. Todas as variáveis tiveram valores observados similares aos expostos por Mochiutti (2007) para a mesma espécie, locais e idades de cultivo.

\section{PCS em função do compartimento de biomassa e idade}

A análise de variância apontou existência de diferença significativa apenas para um dos fatores. O PCS diferenciou-se entre compartimentos de biomassa, ao passo que não houve diferenças significativas para idade e sua interação com compartimento, demonstrando que os fatores são independentes (Tabela 2).

Tabela 2. Análise de variância do PCS da biomassa dos compartimentos de Acacia mearnsii com idades de 1, 3, 5 e 7 anos

\begin{tabular}{lcc}
\hline Fator de variação & GL & QM \\
\hline Idade & 3 & $130.482,37$ ns \\
\hline Compartimento & 3 & $6.673 .895,70^{*}$ \\
Idade $\times$ compartimento & 9 & $70.693,90$ ns \\
\hline Coeficiente de determinação $\left(\mathrm{R}^{2}\right)$ & $38,87 \%$ \\
Coeficiente de variação $(\mathrm{CV} \%)$ & $8,92 \%$ \\
\hline ns = não significativo; ${ }^{*}=$ significativo ao nível de $5 \%$ de \\
probabilidade.
\end{tabular}


As médias, conforme o teste de Tukey ao nível de $5 \%$ de probabilidade, diferenciaram-se apenas nas folhas, que apresentou os maiores valores. O PCS médio para esse compartimento foi de $5.408 \mathrm{kcal} \mathrm{kg}^{-1}$, seguido dos compartimentos casca, galhos e madeira, com, respectivamente, 4.787, 4.692 e $4.572 \mathrm{kcal} \mathrm{kg}^{-1}$ (Tabela 3).

Tabela 3. Médias de PCS da biomassa de madeira, casca, galhos e folhas de Acacia mearnsii

\begin{tabular}{cc}
\hline Compartimento & PCS (kcal kg-1) \\
\hline Folha & $5.408 \mathrm{~A}$ \\
Casca & $4.787 \mathrm{~B}$ \\
Galhos & $4.692 \mathrm{~B}$ \\
Madeira & $4.572 \mathrm{~B}$ \\
\hline
\end{tabular}

Letras minúsculas nas linhas e maiúsculas nas colunas diferem entre si pelo teste de Tukey a $5 \%$ de probabilidade.

Independente dos fatores avaliados, os valores de PCS estão de acordo com os relatados na literatura. Quirino et al. (2005), ao revisarem na literatura o PCS da madeira de 258 espécies florestais, verificaram PCS médio de 4.732 $\mathrm{kcal} \mathrm{kg}^{-1}$, com variação entre 3.350 e 5.260 kcal kg-1.

Para Acacia mearnsii, Silva et al. (2012) apresentam PCS de $4.482 \mathrm{kcal} \mathrm{kg}^{-1}$, ao passo que Vale et al. (2000), ao avaliarem o PCS em diferentes níveis de adubação para Acacia mangium Willd., encontraram valores de PCS entre 4.600 e $4.644 \mathrm{kcal} \mathrm{kg}^{-1}$. Em contrapartida, Pinilla (2000) descreveu o PCS de Acacia mearnsii com valores menores, de 3.494 e $3.993 \mathrm{kcal} \mathrm{kg}^{-1}$.

Ao avaliar a influência etária no potencial dendroenergético, observou-se divergência com os resultados encontrados na literatura. Furtado et al. (2009) avaliaram a influência da idade de Pinus taeda L. na eficiência energética dos resíduos e não constataram diferença significativa, ao passo que Vidaurre et al. (2012) verificaram diferença significativa Schizolobium amazonicum Huber ex Ducke.

Ao avaliar o fator compartimento, os valores obtidos corroboram com a literatura. Eloy et al. (2015b) ao avaliarem o PCS de casca e madeira de Acacia mearnsii encontraram valores menores para madeira quando comparado a casca, com PCS de 4.577 e $4.641 \mathrm{kcal} \mathrm{kg}^{-1}$, respectivamente. Furtado (2012) encontrou valores superiores de PCS de Pinus taeda para os compartimentos acículas e casca.

\section{Estoque de biomassa e produtividade energética total por compartimento de biomassa}

O estoque total de biomassa e a produtividade energética aumentaram com o avanço da idade dos plantios. O estoque de biomassa foi de $3,39 \mathrm{t} \mathrm{ha}^{-1}$ no primeiro ano, 52,81 $\mathrm{t} \mathrm{ha}^{-1}$ no terceiro, 99,71 $\mathrm{t} \mathrm{ha}{ }^{-1}$ no quinto e $135,63 \mathrm{t} \mathrm{ha}^{-1}$ no sétimo ano. A produtividade energética por hectare foi de 16,3; 250,27; 472,54 e $635,56 \mathrm{Gcal}^{-1}$ nos anos 1, 3, 5 e 7, respectivamente (Tabela 4).

Tabela 4. Estoque de biomassa e produtividade energética dos compartimentos de Acacia mearnsii com idades de 1, 3, 5 e 7 anos

\begin{tabular}{ccccccc}
\hline $\begin{array}{c}\text { Idade } \\
\text { (ano) }\end{array}$ & Madeira & Casca & $\begin{array}{c}\text { Galhos } \\
\text { vivos }\end{array}$ & Folhas & $\begin{array}{c}\text { Galhos } \\
\text { mortos }\end{array}$ & Total \\
\hline \multicolumn{7}{c}{ Biomassa (t ha-1 } \\
\hline 1 & 0 & 0 & 2,69 & 0,7 & 0 & 3,39 \\
\hline 3 & 28,36 & 4,79 & 11,39 & 7,45 & 0,82 & 52,81 \\
\hline 5 & 66,26 & 9,75 & 13,04 & 5,72 & 4,94 & 99,71 \\
\hline 7 & 93,64 & 14,75 & 17,65 & 4,92 & 4,67 & 135,63 \\
\hline \multicolumn{7}{c}{ Produtividade energética por unidade de área (Gcal.ha ${ }^{-1}$ ) } \\
\hline 1 & 0 & 0 & 12,56 & 3,73 & 0 & 16,30 \\
\hline 3 & 129,38 & 23,16 & 53,37 & 40,38 & 3,98 & 250,27 \\
\hline 5 & 308,24 & 46,29 & 63,02 & 31,38 & 23,60 & 472,54 \\
\hline 7 & 435,80 & 70,40 & 80,80 & 26,58 & 21,982 & 635,57 \\
\hline \multicolumn{7}{c}{}
\end{tabular}

Garcia et al. (2011), trabalhando com clones de Eucalyptus grandis Hill ex Maiden e Eucalyptus urophylla S. T. Blake, em plantios de 1,5 anos, observaram valores entre 130 e 316,52 Gcal ha-1, dependendo do espaçamento do plantio. De maneira semelhante, para seis espécies de eucalipto aos 3 anos de idade, Brito et al. (1983) encontraram valores entre 662 e $1.259 \mathrm{Gcal} \mathrm{ha}^{-1}$, ao passo que Lima et al. (2011) estimou a produtividade energética de Eucalyptus benthamii aos 6 anos de idade e encontrou $1.940 \mathrm{Gcal} \mathrm{ha}^{-1}$.

\section{CONCLUSÕES}

O poder calorífico superior do compartimento folha é maior e difere dos demais compartimentos de indivíduos de Acacia mearnsii.

Os diferentes compartimentos avaliados da planta, assim como a idade do plantio, induzem em diferenças na produtividade energética da biomassa por área.

A Acacia mearnsii é uma espécie com potencial para suprir a crescente demanda energética por biomassa vegetal.

\section{REFERÊNCIAS}

BRAND, M. A. Energia de biomassa florestal. Rio de Janeiro: Interciencia, 2010. $131 \mathrm{p}$. 
BRASIL. Ministério de Minas e Energia. Balanço Mensal de Energia, 2016. Rio de Janeiro: Ministério de Minas e Energia, 2016. $6 \mathrm{p}$.

BRITO, J. O.; BARRICHELO, L. E. G.; SEIXAS, F.; MIGLIORINI, A. J.; MURAMOTO, M. C. Análise da produção energética e de carvão vegetal de espécies de eucalipto. Boletim Informativo IPEF, v. 23, p. 53-56, 1983.

BYRNE, C. E.; NAGLE, D. C. Carbonization of wood for advanced materials applications. Carbon, v. 35, n. 2, p. 259-266, 1997.

CALDEIRA, M. V. M; RONDOM NETO, R. M.; SCHUMACHER, M. V. Eficiência do uso de micronutrientes e sódio em três procedências de acácia-negra (Acacia mearsii De Wild.). Revista Árvore, v. 28, n. 1, p. 39-47, 2004.

DOLCl, M.; SANQUeTTA, C. R.; CORTE, A. P. D.; BEHLING, A.; RODRIGUES, A. L.; SIMON, A. A. Equações para estimativa de biomassa total ao longo da rotação de povoamentos de acácianegra. In: Congresso Latino-Americano de IUFRO. San José. Anais... San José: IUFRO, 2013.

ELOY, E. CARON, B. O.; SILVA, D. A.; SOUZA, V. Q.; TREVISAN, R.; BEHLING, A.; ELLI, E. F. Produtividade energética de espécies florestais em plantios de curta rotação. Ciência Rural, v. 45, n. 8 , p. $1424-1431,2015 a$.

ELOY, E.; SILVA, D. A.; CARON, B. O.; SOUZA, V. Q. Capacidade energética da madeira e da casca de acácia-negra em diferentes espaçamentos. Pesquisa Florestal Brasileira, v. 35, n. 82, p. 163$167,2015 b$.

FURTADO, T. S.; FERREIRA, J. C.; NEVES, M. D.; BRAND, M. A. Influência da idade da árvore na eficiência energética dos resíduos de Pinus taeda. In: Congresso brasileiro sobre florestas energéticas, Belo Horizonte. Anais... Colombo: Embrapa florestas, 2009.

FURTADO, T. S.; FERREIRA, J. C.; BRAND, M. A.; NEVES, M. D. Correlação entre o teor de umidade e eficiência energética de resíduos de Pinus taeda em diferentes idades. Revista Árvore, v. 36, n. 3, p. 577-582, 2012.

GARCIA, E. A.; LANÇAS, K. P.; GUERRA, S. P. S.; REZENDE, M. A.; MAZIERO, F. L. A energia da madeira em floresta de eucalipto. In: Congresso Brasileiro de Engenharia Agrícola, Cuiabá. Anais... CONBEA, 2011.

KÖPPEN, W.; GEIGER, R. Klimate der Erde. Gotha: Verlag Justus Perthes. 1928. Wall-map $150 \mathrm{~cm} X 200 \mathrm{~cm}$.

LIMA, E. A.; SILVA, H. D.; LAVORANTI, O. J. Caracterização dendroenergética de árvores de Eucalyptus benthamii. Pesquisa Florestal Brasileira, v. 31, n. 65, p. 09-17, 2011.

MOCHIUTTI, S. Produtividade e sustentabilidade de plantações de acácia-negra (Acacia mearnsii De Wild.) no Rio Grande do Sul. 2007. 270f. Tese (Doutorado em Ciências Florestais) Universidade Federal do Paraná, Curitiba, 2007.

PARIKH, J.; CHANNIWALA, A. S.; GHOSAL, G. K. A correlation for calculating HHV from proximate analysis of solid fuels. Fuel, v. 84, n. 5 , p. $487-494,2005$.
PINILLA, J. C. Descripción y antecedentes básicos sobre Acacia dealbata, A. melanoxylon y A. mearnsii. Conception, Chile: INFOR-CORFO, 2000. 49 p. (Informe Técnico, 147).

QUIRINO, W. F.; VALE, A. T.; ANDRADE, A. P. A.; ABREU, V. L. S.; AZEVEDO, A. C. S. Poder calorífico da madeira e de materiais lignocelulósicos. Revista da Madeira, v. 89, p. 100-106, 2005.

SAS LEARNING EDITION. Getting started with the SAS Learning Edition. Cary, 2003. 200 p.

SFB - Serviço Florestal Brasileiro. Florestas do Brasil em resumo: dados de 2007 - 2012. Brasília: SFB, 2013. 188 p.

SILVA, D. A.; CARON, B. O.; BEHLING, A.; SOUZA, V. Q.; ELOY, E. Ponto de amostragem ao longo do fuste para estimativa do poder calorífico da madeira. Ciência Rural, v. 42, n. 9, p. 15881595, 2012.

VAle, A. T.; BRASIL, M. A. M; CARVAlho, C. M.; VeigA, R. A. A. Produção de energia do fuste de Eucalyptus grandis e Acacia mangium em diferentes níveis de adubação. Cerne, v. 6, n. 1 , p. $83-88,2000$.

VIDAURRE, G. B.; CARNEIRO, A. C. L.; VITAL, B. R.; SANTOS, R. C.; VALLE, M. L. A. Propriedades energéticas da madeira e do carvão de paricá (Schizolobium amazonicum). Revista Árvore, v. 36, n. 2, p. 365-371, 2012. 